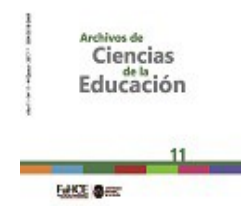

Archivos de Ciencias de la Educación, Vol. 11, nº 11, 2017, e018. ISSN 2346-8866

Universidad Nacional de La Plata.

Facultad de Humanidades y Ciencias de la Educación.

Departamento de Ciencias de la Educación.

\title{
Introducción. Reflexiones en torno a la historia y al presente de la Didáctica general
}

\author{
Introduction. Reflections on the history and present of the general \\ Didactics.
}

\section{Sofía Picco *}

* Universidad Nacional de La Plata, Argentina | sofia.picco.s@gmail.com

La Didáctica general es una disciplina que posee un largo y rico recorrido histórico así como también una vasta y diversa producción en el presente. Se ocupa desde su surgimiento de estudiar las prácticas de enseñanza y de colaborar con los docentes en la resolución de los problemas a los que se enfrenta en su trabajo cotidiano.

La Didáctica tiene vigencia y puede brindar diversos aportes conceptuales e instrumentales para el mejoramiento de las prácticas de enseñanza. El campo ha ido creciendo y se ha ido modificando, ha atravesado por diferentes procesos, ha establecido vínculos con distintas disciplinas y ha recibido varios cuestionamientos poniendo en discusión su legitimidad.

En este sentido, resulta oportuno generar desde Archivos de Ciencias de la Educación un espacio de visualización de algunas producciones actuales, de análisis de problemáticas teórico-prácticas que atraviesan la Didáctica y de reflexión epistemológica, entendiéndola como un ejercicio meta-teórico crítico sobre la propia disciplina, sus límites y posibilidades actuales de construcción.

En los últimos 20 años, diferentes autores han revisado cuál es el objeto de estudio de la Didáctica, a saber: la enseñanza, el aula de clases en donde ésta se desarrolla prioritariamente, el proceso de enseñanzaaprendizaje, etc. También otros teóricos han discutido acerca de su legitimidad como disciplina, por ejemplo, para algunos la Didáctica ha perdido vigencia o debe ser reemplazada por el valioso aporte de las Didácticas 
especiales, principalmente constituidas en torno a los contenidos específicos de enseñanza, o debe ser sustituida por el Curriculum, considerado un objeto de estudio de la Didáctica o como otro campo que se ha ido fortaleciendo. Entre aquellos autores que siguen sosteniendo la existencia de la Didáctica se generan diferentes posiciones en torno a si ésta debe evidenciar un carácter básicamente descriptivo y explicativo comprensivo ${ }^{\underline{1}}$, cuyo vínculo con la práctica sea indirecto y quede librado al uso y a la interpretación que los docentes hagan de las teorías didácticas, o si por el contrario la Didáctica tiene que asumir un mayor compromiso con las prácticas de enseñanza, fortaleciendo aquella dimensión normativa que la acompaña desde su surgimiento.

Aunque se pueden configurar otras aristas que evidencian la necesidad de una reflexión epistemológica en la Didáctica como, por ejemplo, las complejas relaciones interdisciplinarias que caracterizan al conjunto de las Ciencias Sociales, este Dossier pretende abrir líneas de análisis en torno a las problemáticas antes enunciadas. En este sentido, las autoras que dan vida a esta compilación profundizan en sus artículos en aspectos nodales del campo de la Didáctica y abren, como parte de la dinámica propia del conocimiento científico, líneas en las cuales seguir reflexionando y construyendo conocimientos.

La primera contribución proviene de María Esther Aguirre Lora, quien nos brinda desde la Universidad Autónoma de México una mirada renovada sobre la Didáctica Magna, considerada la obra más conocida de Juan Amós Comenio y el hito fundacional de la Didáctica.

En primer lugar, la autora contextualiza la obra comeniana en los tiempos modernos, configurando un entramado complejo que permite entender aquella articulación, hoy añorada, entre explicación, norma y utopía con la que Gimeno Sacristán en 1978 caracterizaba la constitución epistemológica de las Ciencias de la Educación y luego Davini en 1996, en nuestro medio, resignificaba como propia de la Didáctica.

La dimensión operativa de la Didáctica, como la conceptualiza la autora, (y que constituye un hilo de articulación entre los tres artículos de este Dossier) no puede pensarse al margen de la concepción de la naturaleza y de la cosmovisión religiosa del mundo que sustentaba Comenio. En este sentido, la norma comeniana expresaba la forma en la que debían hacerse las cosas, tal como indica una de las acepciones de norma (Diccionario de la Real Academia Española, 2001). Si se sigue la norma, se podrá alcanzar la concreción de un modelo ideal que sirve, a su vez, de patrón de comparación para la evaluación de las acciones.

Entonces, este trabajo de Aguirre Lora llama la atención sobre el componente normativo de la Didáctica, con el que nace la disciplina y que hoy se encuentra en un profundo proceso de revisión. A su vez, la pertinencia de este trabajo radica en resignificar un aspecto central de la obra comeniana y de la reflexión didáctica actual: la enseñanza como un arte. Ese saber hacer que ocupaba y preocupaba a Comenio, está también presente en las últimas producciones del campo de la formación docente.

Por último, este trabajo habilita, entre otras, las siguientes preguntas: ¿la Didáctica se pregunta hoy cómo enseñar todo a todos, como decía Comenio, o cómo ayudar a que muchos enseñen en el marco de los grandes sistemas educativos? (Feldman, 1999, 2002; Terigi, 2006) ¿Cómo se configura la dimensión normativa en la Didáctica en un contexto posmoderno, con el fracaso de las utopías modernas, la crisis de valores y del sistema educativo más específicamente, y el debilitamiento del discurso y las propuestas de la pedagogía, por mencionar algunos de los procesos extra-didácticos que considera Davini (2008)? ¿Cómo podemos configurar hoy aquella tensión/convivencia entre arte y ciencia en la enseñanza que destaca Aguirre Lora en la obra de Comenio y que está presente en otros pensadores, filósofos y pedagogos?

La segunda contribución a este Dossier la realiza Victoria Baraldi desde la Universidad Nacional del Litoral (Argentina), quien plantea una reflexión en torno a la enseñanza de la Didáctica en la formación de profesores universitarios. 
La Didáctica es una disciplina que se ocupa de estudiar las prácticas de enseñanza y en este proceso es central el diálogo que mantiene con el docente. Además, ha estudiado diversos objetos que le incumben como, por ejemplo: curriculum, evaluación, formación docente, estrategias didácticas, programación, etc. Construir saberes teóricos y prácticos en torno a estas preocupaciones confiere actualidad al conocimiento didáctico.

Específicamente, la formación de profesores universitarios, en torno a la cual Baraldi estructura su trabajo, es una problemática didáctica, pero también curricular, pedagógica, institucional, de política educativa y presente en la sociedad. La complejidad de la formación docente demanda de la Didáctica la construcción de una perspectiva multirreferencial (Souto, 1996; Ardoino, 2005) que articule líneas teóricas diversas para poder brindar categorías conceptuales e instrumentales para intervenir en la enseñanza en un segundo orden, es decir: para ayudar a los docentes a enseñar pero también para conceptualizar la enseñanza como contenido que intermedia la relación didáctica en los espacios de formación docente.

A este entramado disciplinar se adiciona la complejidad de pensar su enseñanza. ¿Cómo y qué enseñar de Didáctica a los futuros profesores universitarios? Aparecen de esta manera muchas preguntas desde la propia disciplina y la formación docente que el trabajo de Baraldi ayuda a enriquecer, entre ellas: ¿qué saberes, herramientas, capacidades, debe poseer hoy un docente para enfrentar desde el aula y con centralidad en la enseñanza, otros múltiples procesos que lo atraviesan a él y a sus estudiantes? ¿Qué hay que saber hoy para saber enseñar o cuáles son en la actualidad los “requerimientos epistémicos de la enseñanza”, como analiza Jackson (2002)? ¿Cómo puede la Didáctica ayudar a los docentes a enseñar? ¿Cómo construir desde una cátedra de “Didáctica general” la formación de profesores intelectuales, críticos, reflexivos?

El tercer trabajo que integra esta selección le corresponde a Ana Lía Cometta, perteneciente a la Universidad Nacional de San Luis (Argentina). La autora reafirma el carácter propositivo de la Didáctica que, como dije, acompaña a la disciplina desde su surgimiento. La dimensión operativa -Aguirre Lora-, el carácter propositivo -Cometta-, la base normativa -Davini (2008) -, integra la constitución epistemológica de la Didáctica como disciplina.

Tal vez, la pregunta que haya que hacerse a partir de aquí refiera al carácter de esa normatividad y al lugar que ocupan en ella los saberes de los docentes. En primer lugar, es interesante la caracterización que realiza Frigerio cuando expresa que una norma debe poseer una "textura abierta” (1991, p.27). Así, la norma didáctica podría representarse como una red en cuyos intersticios emergen la libertad y creatividad del docente, necesarias para su resignificación y para la apertura de aquellos espacios indeterminados de la práctica. Parafraseando a la autora, no hay normativa que pueda clausurar todos los significados que le pueden adjudicar los sujetos.

En segundo término: ¿qué lugar ocupan los saberes de los docentes, las experiencias acumuladas en la enseñanza, en la construcción de ese carácter propositivo? ¿Cómo se integra la relación necesaria entre conocimiento y acción del docente en el corpus de la Didáctica? ¿Cómo se entiende el razonamiento práctico del docente, como analiza Fenstermacher (1989), y cómo se influye en él para impactar realmente en las prácticas de enseñanza? Estas preguntas de actualidad y relevancia, encuentran en el artículo de Cometta un punto indispensable de reflexión, sobre todo a partir de la apuesta que realiza la autora al "valor de uso del conocimiento didáctico” para el análisis y la toma de decisiones en las prácticas de enseñanza.

Volviendo al comienzo, espero que la lectura de estos trabajos enriquezca la reflexión en el campo de la Didáctica y contribuya a pensar y a concretar más y mejores prácticas de enseñanza para todos. 


\section{Notas}

1 Las explicaciones comprensivistas apuntan a dar cuenta de la multiplicidad de dimensiones que intervienen en la situación a la que esperan darle inteligibilidad, además de los sentidos que los sujetos sociales como tales les asignan a sus acciones (Klimovsky e Hidalgo, 1998).

\section{Bibliografía}

Ardoino, J. (2005). Complejidad y formación. Pensar la educación desde una mirada epistemológica. Buenos Aires: Novedades Educativas.

Davini, M. C. (1996). La formación docente en cuestión: política y pedagogía. Buenos Aires: Paidós.

Davini, M. C. (2008). Métodos de enseñanza. Didáctica general para maestros y profesores. Buenos Aires: Santillana.

Diccionario de la Lengua Española de la Real Academia Española. (2001). 22a edición. Disponible en http://www.rae.es/rae.html

Feldman, D. (1999). Ayudar a enseñar. Relaciones entre didáctica y enseñanza. Buenos Aires: Aique.

Feldman, D. (2002). Reconceptualizaciones en el campo de la didáctica. En Gonçalves Rosa, D. y de Souza, V. C. (Orgs.). Didática e práticas de ensino: interfaces com diferentes saberes e lugares formativos (pp. 6984). Rio de Janeiro y Goiania: DP\&A Editora y Editora Alternativa.

Fenstermacher, G. (1989). Tres aspectos de la filosofía de la investigación sobre la enseñanza. En Wittrock, M. La investigación de la enseñanza, I. Enfoques, teorías y métodos (Vol. I, pp. 149-179). Madrid: Centro de Publicaciones Ministerio de Educación y Cultura y Paidós Ibérica.

Frigerio, G. (1991). Curriculum: norma, intersticios, transposición y textos (capítulo 1). En Frigerio, G. (Comp.), Curriculum presente ciencia ausente. Normas, teorías y críticas (Vol. I, pp.17-45). Buenos Aires: Miño y Dávila; FLACSO.

Gimeno Sacristán, J. (1978). Explicación, norma y utopía en las ciencias de la educación. En Escolano, A. Epistemología y educación (pp.158-167). Salamanca: Sígueme.

Jackson, P. (2002). Práctica de la enseñanza. Buenos Aires: Amorrortu.

Klimovsky, G. e Hidalgo, C. (1998). La inexplicable sociedad. Cuestiones de epistemología de las ciencias sociales. Buenos Aires: A-Z Editora.

Souto, M. (1996). La clase escolar. Una mirada desde la didáctica de lo grupal. En: Camilloni, A. et al. Corrientes didácticas contemporáneas (pp.117-155). Buenos Aires: Paidós.

Terigi, F. (2006). Tres problemas para las políticas docentes. En Encuentro Internacional La docencia, ¿una profesión en riesgo? Condiciones de trabajo y salud de los docentes. Organizado por la OREALC, Oficina Regional de Educación de la UNESCO para América Latina y el Caribe. Montevideo, 22, 23 y 24 de junio. Recuperado de: www.oei.es/historico/docentes/articulos/tres problemas politicas docentes terigi.pdf 